\title{
High-Energy Phenomena in Galaxy Clusters
}

\author{
Sergio Colafrancesco*† \\ School of Physics, Wits University, Johannesburg, South Africa \\ sergio.colafrancescodwits.ac.za
}

\section{Paolo Marchegiani}

School of Physics, Wits University, Johannesburg, South Africa

paolo.marchegianidwits.ac.za

Galaxy clusters are the largest storage rooms for cosmic material (galaxies, dark matter, hot thermal baryonic plasma, non-thermal and relativistic plasma, black holes, magnetic fields, cosmic rays). In this sense they can be considered as the largest multi-disciplinary laboratories in the universe where one can efficiently study some of the most interesting aspects of the astrophysics of large-scale structures: the nature of dark matter, the origin and distribution of cosmic rays, the impact of magnetic fields on large-scale structures, the impact of black holes on large-scale structures, the interplay between thermal and non-thermal phenomena. As such, galaxy clusters are environments for the production of high-energy emission from the cluster diffuse medium. Many theoretical expectations have been produced but no clear evidence of high-E emission (at $E>20 \mathrm{keV}$ ) has been obtained so far. We discuss in this paper the main results and controversies posed by theoretical and observational challenges on the physics of galaxy clusters by using a multi-frequency analysis.

XI Multifrequency Behaviour of High Energy Cosmic Sources Workshop

25-30 May 2015

Palermo, Italy

\footnotetext{
* Speaker.

${ }^{\dagger}$ Contact author.
} 


\section{Phenomenology}

Galaxy clusters, the largest gravitationally bound structures in the universe, are representative systems of the distribution of large-scale structures (LSS) in the universe. The description of these systems is continuously enriching of physical details regarding their matter and field content. This in turn opens the full multi-frequency window to the analysis of both thermal and non-thermal phenomena and prefigures a rich phenomenology of high-E emission from these structures.

Other than the galaxies visible in the cluster, several evidences show that diffuse components exist in the intra cluster medium (ICM).

Dark Matter (DM) is the dominant form of matter that creates the potential wells of galaxy clusters and all LSS. If we consistently take into account the fundamental nature of DM particles, we are inevitably bound to consider the effects of their annihilation or decay on the structure and evolution of the DM clumps (see Colafrancesco 2010).

Baryonic material collected in the DM potential wells of galaxy clusters is mainly present as a diffuse hot plasma (the intra-cluster medium, ICM) visible in X-rays (see, e.g., Sarazin 1988). During the history of the cluster evolution, this plasma is likely heated and shocked by large-scale shock waves produced in continuous accretion and/or violent merging processes, as found in cosmological simulations (see, e.g, Ryu et al. 2003), producing a complex distribution of Mach numbers. The occurrence of shock waves naively suggests that Fermi-like acceleration might take place in galaxy clusters thereby accelerating cosmic rays (CRs) that can be efficiently confined in the cluster atmosphere (Colafrancesco et al. 2006). Radio halos and relics are the most evident signals of the presence of these non-thermal diffuse component in galaxy clusters.

Radio observations and magneto-hydrodinamic (MHD) simulations also suggest that magnetic fields are associated to the distribution of baryonic material collected in the cluster potential wells. The seed magnetic field is likely amplified and made turbulent by the coupling of gravitational collapse and MHD processes during cluster formation. Evidence for wide-scale and turbulent intracluster magnetic field is indicated by the diffuse radio synchrotron emission found in many clusters (with typical values $B \sim 0.1-2 \mu \mathrm{G}$, derived from equipartition assumption), by radio relics in dynamically active clusters (with typical values $B \sim 0.2-5 \mu \mathrm{G}$ ) and by Faraday rotation measurements of background and embedded polarized radio sources (with typical values $B \sim 1-50 \mu \mathrm{G}$; Govoni \& Feretti 2004).

Very massive DM clumps that collapse at high redshifts $(z \sim 6-7)$ often contain the most massive black holes (BHs) at their centers (see the Millennium simulation: Springel et al. 2005). The AGN descendants of these ancient supermassive BHs (SMBHs) are found to be part of the massive galaxies located at the centers of the most massive galaxy clusters we observe at the present epoch, like, e.g., CenA, M87/Virgo, NGC1275/Perseus, A262, A4059, with their radio lobes penetrating the ICM for tens or hundreds of kpc. It is often observed that the radio jets/lobes end up in approximately spherical bubbles of relativistic plasma (likely containing relativistic or mildlyrelativistic particles) that appear as cavities in the X-ray images of galaxy clusters (Birzan et al. 2004 ) with dimensions ranging from a few $\mathrm{kpc}$ (as in Perseus) to $\sim 100 \mathrm{kpc}$ (as in the case of the cluster MS0735-556). The combination of high-resolution radio and X-ray images indicates that the relativistic plasma found inside the X-ray cavities is connected with the jet/lobe structure of the central AGN and with the history of the ejection events and mechanisms from the central AGN. 
The previous evidence indicates that galaxy clusters are the largest storage rooms for cosmic material (galaxies, DM, hot thermal baryonic plasma, non-thermal and relativistic plasma, BHs, magnetic fields, CRs). In this sense they can be considered as the largest multi-disciplinary laboratories in the universe where one can efficiently study some of the most interesting aspects of the astrophysics of LSS: the nature of DM, the origin and distribution of CRs, the impact of magnetic fields on LSS, the impact of BHs on LSS, the interplay between thermal and non-thermal phenomena in LSS. As such, galaxy clusters are also environments for the production of high-E emission from both the cluster diffuse medium and embedded discrete sources. Many theoretical expectations have been produced but no clear evidence of high-E emission (at $E>20 \mathrm{keV}$ ) has been obtained so far.

In the following we will discuss the main results and controversies posed by theoretical and observational challenges on the physics of galaxy clusters by using a multi-frequency analysis.

\section{Astro-physical controversies}

There are several theoretical and observational controversies about the origin of the nonthermal (high-E) phenomena in galaxy clusters and we list below the main ones.

\subsection{The cooling-flow problem}

Theoretical description of the ICM in the central regions of many clusters predicts that it is cooler than in their outskirts, because it radiates X-rays at such a rate that the plasma cooling time is much shorter than the age of the cluster. However, such rapid cooling is not observed and the central-temperature depression in cluster cores is not as deep as expected on the basis of the plasma's cooling rate: the observed central temperature $T_{\text {inner }}$ settles, in fact, at a fraction $\sim 1 / 3-1 / 2$ of the outer temperature $T_{\text {outer }}$. The lack of cooling gas with $T<T_{\text {inner }}$ in cluster cores is also supported by the absence of line emission corresponding to gas below $T_{\text {inner }}$, as shown by high spatial resolution imaging with Chandra and high spectral resolution measurements with the XMM-Newton RGS instrument (see, e.g., Colafrancesco \& Marchegiani 2008). It has been widely recognized that some form of heating is necessary to quench the cooling flow $(\mathrm{CF})$ and form a warm-core in the IC gas structure. Available sources of heating in cluster cores are provided by AGN jets and lobes, pressure waves, buoyant bubbles and cavities, shock waves, leptonic and hadronic cosmic-rays. All of these heaters imply a connection of CFs with high-E processes in clusters.

Some difficulties with solutions along these lines, however, might occur: a first difficulty is that not all cooling flow clusters contain a powerful radio source at their center (see, e.g., the cluster RXJ0820.9+0752, Bayer-Kim et al. 2002; and the clusters A1650 and A2244, Donahue et al. 2005), nor a large amount of spherically distributed buoyant bubbles out to the cluster's core boundary. Another difficulty is that the bremsstrahlung cooling rate is proportional to $n_{e}^{2} T^{1 / 2}$, with $n_{e}$ the plasma's electron number density and $T$ its local temperature: a suitable conduction and/or heating and pressure-building mechanism must somehow adapt itself to this behaviour, particularly as a function of $n_{e}(r)$, which varies by orders of magnitude along the clusters' radii. Other difficulties stand out with the spatial distribution of the energy deposited by AGN jets and cavities: on one hand, the energy released from AGN jets and cavities seems to be sufficient to quench cooling flow 
and increase the central gas entropy (e.g., Sijacki \& Springel 2006); on the other hand, it is not yet clear if such energy can be properly distributed to reproduce the observed cool-core temperature profiles (see, e.g., discussion by Vernaleo \& Reynolds 2005, 2006; Heinz et al. 2006; Vernaleo \& Reynolds 2007). Small duty cycles and a higher fraction of AGNs in cluster cores (see Bird et al. 2008) might however weaken the difficulties related to the AGN heating scenarios. In addition, there seems to be a small AGN activity in groups (Dwarakanath \& Nath 2006). Sound waves and shocks do not seem to be efficient in quenching cooling flows (see, e.g., Fujita \& Suzuki 2005).

Irrespective of the nature of the heating source, it seems that a general property of the heating agents - which derives from the smoothness and from the similarity of the heating distribution required to quench cooling in several clusters - is that they must be spatially distributed within the whole cluster core. In fact, it has been shown (Fabian et al. 2006; Fabian \& Sanders 2007; Sanders \& Fabian 2007) that the non-thermal pressure in the Perseus cluster (one of the best studied clusters with cool cores and mini radio-halos) is quite similar to (but slightly steeper than) the thermal one. In this context, it has been shown that a CR population in clusters that is radially distributed similarly to the radial profile of the IC plasma, and are acting as Warming Rays, is able to recover the observed radial structure of the IC plasma temperature in several clusters (Colafrancesco et al. 2004, Colafrancesco \& Marchegiani 2008). However, recent upper limits from Fermi-LAT have shown that in some cluster the amount of non-thermal protons is not sufficient to solve completely the cooling flow problem (e.g. Huber et al. 2013), therefore the action of some other source of heating is necessary.

Detection of high-E emission from cluster cores will allow to unveil the nature of the heating mechanism and associate it to specific structures (e.g., active galaxies, shocks, etc.) within the cluster core. The presence of CRs in cluster cores has been also related to the existence of radio mini halos, suggesting the possibility that quenching of cooling-flows is related to non-thermal cosmic rays (Gitti et al. 2002), thus connecting the low- $v$ and high-E phenomenology of nonthermal processes.

\subsection{Origin of radio halos and relics}

In the radio frequency band, detection of giant radio halos and relics is the most evident indication of the presence of diffuse non-thermal components in galaxy clusters, i.e. relativistic electrons and magnetic fields that produce radio emission by synchrotron emission (see, e.g., Feretti et al. 2012). At present, these emissions are observed in about 100 clusters. Usually, diffuse radio emission features in galaxy clusters are classified as:

- radio halos $(\mathrm{RH})$ : located close the center of the cluster, with a regular morphology, size of the order of Mpc and with low radio polarization level;

- radio mini-halos $(\mathrm{RmH})$ : located close the center of cool-core clusters, with size $\lesssim 500 \mathrm{kpc}$;

- radio relics (RR): usually located in peripheral regions of clusters, with irregular shape and higher level of radio polarization.

These observations imply that relativistic electrons and magnetic fields must be present on extended scales. Since electrons with Lorentz factors $\gamma \sim 10^{3}$ have lifetimes $\sim 10^{8}$ yrs (se, e.g., Sarazin 1999), and on this time scale their travel distance in a magnetized medium is of the order of $50 \mathrm{kpc}$ (e.g. Blasi \& Colafrancesco 1999), some process that produces or accelerates relativistic electrons on larger scale must be present. For radio halos, several models have been pro- 
posed to solve this problem: primary electrons, i.e. accelerated at IC shocks (Tribble 1993) or injected by galaxies (Jaffe 1977), electrons re-accelerated by IC turbulences (Brunetti et al. 2001), secondary electrons produced in hadronic interactions (Dennison 1980; Blasi \& Colafrancesco 1999), secondary electrons produced in Dark Matter annihilations (Colafrancesco \& Mele 2001; Colafrancesco et al. 2006).

For radio relics, the common explanation is that they are related to the acceleration produced by shocks occurring during merging processes. However, several issues are still debated in this context. Direct acceleration or re-acceleration of electrons results to be very inefficient, producing a strong heating of the IC plasma (Wolfe \& Melia 2006, Petrosian \& East 2008), and requires strong fine-tuning to explain the available observations; simple hadronic secondary models cannot explain the high-frequency steepening of the radio halo spectrum observed in some clusters (see, e.g., Thierbach et al. 2003) and, more critically, they predict a strong gamma-ray emission that has not been observed in galaxy clusters so far (e.g. Ackermann et al. 2014); also Dark Matter secondary models predict a gamma-ray emission, and depends on the physical properties of Dark Matter that are currently unknown. To address these issues, hybrid models have been proposed, like the re-acceleration of secondary electrons of hadronic origin (Brunetti et al. 2012), or mixed secondary and primary electron models dominating the diffuse radio emission in different regions of the cluster (see Zandanel et al. 2014).

Therefore, more detailed observations and models are necessary to solve these problems. At present, we can identify some open issues that every model must explain:

- many clusters with ongoing merging do not host a radio halo: therefore it is necessary to explain why in some clusters a merging produces a radio halo and in other clusters it does not do it;

- radio halos clusters apparently follow a correlation between their radio $L_{R}$ and X-ray $L_{X}$ luminosity (see, e.g., Cassano et al. 2013), and there is an apparent bi-modality between radio quiet and radio loud clusters; however, this correlation is quite scattered, there are some cluster which are outside this correlation (Govoni et al. 2012), and some recent observations have discovered some clusters that seem to fill the gap between radio loud and radio quiet clusters (Bonafede et al. 2015; Bernardi et al. 2016; Knowles et al. 2016), or have radio luminosities upper limit far below the limits of other radio quiet clusters (George et al. 2015); therefore the real distribution of clusters in the $L_{R} / L_{X}$ plot could be more complex than the claimed bimodal one;

- in some clusters the spatial distribution of the radio halos is more similar to the distribution of the galaxies rather than to the distribution of the thermal X-ray gas (Govoni et al. 2012), and in some cases it is similar also to the distribution of Dark Matter (see Brown \& Rudnick 2011 for the case of Coma). In some cases, like the Bullet cluster, the radio halo distribution has peaks that are close to both the X-ray peaks and the Dark Matter peaks (Marchegiani \& Colafrancesco 2015);

- radio relics often are displaced from merging shock fronts (e.g. Brown \& Rudnick 2011), have low Mach number $(M \sim 1-3)$ and the standard acceleration processes are inefficient to produce these radio structures (Vazza \& Bruggen 2014).

It is clear that the explanation of these emission phenomena (both radio halos and relics) are still open and more detailed models and multi-frequency observations (especially in radio, hard $\mathrm{X}$-ray, and gamma-ray bands) are necessary to solve them. 


\subsection{The amount of cosmic rays}

Related to the problem of the origin of CRs is the determination of their whole amount in galaxy clusters. In fact, the presence of RHs in clusters requires an additional non-thermal pressure (energy density) component in addition to the thermal pressure (energy density) provided by the ICM. Attempts to determine the amount of non-thermal pressure compared to the thermal have been done using a multi-frequency analysis. It has been recognized that galaxy clusters hosting RHs show a correlation between their radio power measured at $1.4 \mathrm{GHz} P_{1.4}$ due to synchrotron emission, and their X-ray luminosity $L_{X}$ due to thermal bremsstrahlung emission (see, e.g., Colafrancesco 1999, Giovannini et al. 1999, Feretti et al. 2012) that can be fitted with a power law $P_{1.4} \propto L_{X}^{d}$ with slope $d$ lying in the range 1.5 to 2.1 (see, e.g., Brunetti et al. 2009 for a recent compilation). Such a correlation links the non-thermal particle and magnetic field energy density (pressure) related to the synchrotron radio luminosity $P_{1.4} \propto n_{e, \text { rel }} B^{(\alpha+1) / 2} v^{-(\alpha-1) / 2} \sim P_{n o n-t h} U_{B}^{(\alpha+1) / 4}$ (where $\alpha$ is the slope of a power-law electron spectrum $n_{e, r e l} \sim E^{-\alpha}$ ), with the thermal pressure $P_{t h}$ of the ICM, related to the thermal bremsstrahlung X-ray emission given by $L_{X} \propto n_{e}^{2} T^{1 / 2} \sim P_{t h} t_{c o o l}^{-1}$, where $P_{t h} \propto n_{e} T$ and $t_{\text {cool }} \propto T^{1 / 2} n_{e}^{-1}$.

An analogous correlation has been found (see, e.g., Basu 2012, Colafrancesco et al. 2014) between $P_{1.4}$ and the integrated Compton parameter $Y_{S Z}$ due to the Sunyaev-Zel'dovich effect (SZE) produced by inverse Compton scattering of CMB photons off the electron populations which are residing in the cluster atmosphere (see Colafrancesco et al. 2003 for details, and Colafrancesco 2013 for a recent review). The Compton parameter $Y_{S Z} \propto \int d \ell P_{t o t}$ is proportional to the total particle pressure (energy density) provided by all the electron populations in the clusters atmosphere (see Colafrancesco et al. 2003): the cluster atmosphere is thus the combination of the thermal plasma producing $X$-ray emission and the non-thermal plasma, at least the one producing synchrotron radio emission. Therefore, the $P_{1.4}-Y_{S Z}$ correlation links the non-thermal particle and B-field pressures, as measured by $P_{1.4}$, with the total particle pressure $P_{t o t}$, as measured by $Y_{S Z}$. For the sake of generality we express the total particle pressure as $P_{t o t}=P_{t h}+P_{n o n-t h}=P_{t h}[1+X]$ where $X \equiv P_{\text {non-th }} / P_{t h}$.

From the analysis of the $Y_{S Z}-L_{X}$ correlation, a non-thermal to thermal pressure ratio of the order of $P_{n o n-t h} / P_{t h} \sim 0.55$, has been estimated (Colafrancesco et al. 2014; see Figure $\mathbb{W}$ ), therefore requiring a strong source of particle injection or acceleration. However, recent progresses in this field have shown that acceleration mechanisms by shocks or turbulences are inefficient and mainly heat the ICM (Wolfe \& Melia 2006, Petrosian \& East 2008). In addition, the CR content in clusters is strongly limited by recent Fermi-LAT upper limits on gamma-ray emission (Vazza et al. 2016).

A key question to understand the acceleration processes in the ICM concerns the energy spectrum of the emitting particles. Synchrotron emission depends in a degenerate fashion on the magnetic field energy and on the energy of the CRe. Hence, in order to disentangle the energy of the emitting particles from the synchrotron observations, we need both constraints on the magnetic field and on the spectrum of the radio emission over the largest possible frequency range.

\subsection{B-field: origin and amplitude}

Magnetic fields are ubiquitous in the universe but their origin is still unknown. On large scales, magnetic fields are the hardest to explain because the usually invoked dynamo mechanism does not 


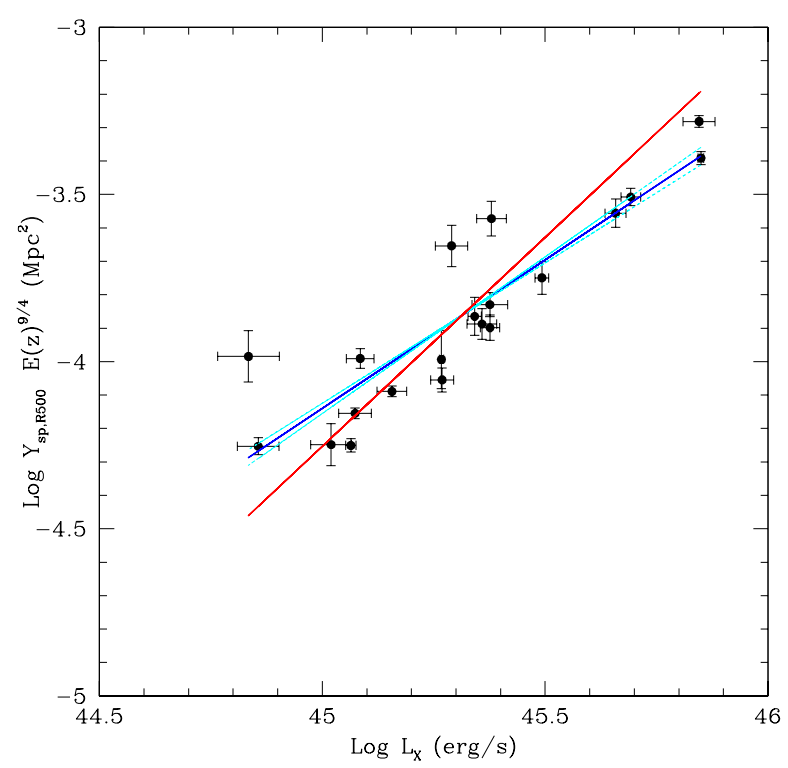

Figure 1: $Y_{S Z}-L_{X}$ relation in a sample of 23 clusters; best fit line (solid blue) together with the associated uncertainties in the slope and intercept (dashed cyan) and the theoretical expectation (solid red) for the a simple hydrostatic equilibrium in all clusters are shown. The best-fit value of $X$ is $0.55 \pm 0.05$. Figure from Colafrancesco et al. (2014).

have the time to amplify the field on the largest scales starting from a weak initial seed. In addition, the magnetic fields in galaxy clusters are poorly constrained from an observational point of view, and it is unclear whether they are formed by primordial seeds - amplified during the process of structure formation - or are formed by magnetic fields seeded by AGN or galactic outflows. The presence of magnetic field in galaxy clusters can be probed by diffuse radio sources associated with clusters (see Johnston-Hollitt et al. 2015, Govoni et al. 2015, Ferrari et al. 2015), and by Faraday rotation measures (RM) of embedded and background polarized sources (see e.g. Govoni \& Feretti 2004 for a review). In the last decade, there have been revolutionary improvements in modelling the magnetic field in galaxy clusters, based partly on observations and partly on numerical simulations (e.g. Bonafede et al. 2011, Bruggen 2013, Dolag et al. 2008, Govoni et al. 2006, Vacca et al. 2012). Much of what is known about the magnetic field in galaxy clusters comes from sensitive polarized observations. The observed polarization angle of a synchrotron-emitting source observed in the background of a galaxy cluster is rotated with respect to the intrinsic polarization angle by the Faraday Rotation Measure defined as $R M=\int_{\text {cluster }} n_{e}(l) B_{\|}(l) d l$, where $n_{e}$ is the thermal gas density in the ICM, and $B_{\|}$is the magnetic field component along the line-of-sight. Recent studies have given us important clues to elucidate the evolution of the magnetic fields in galaxy clusters. However, because of the limits of current instruments, further improvements will be hard to achieve. A primary limiting factor is the sensitivity of present facilities, that limits the feasibility of such studies to a few nearby clusters where a sufficient number of background and embedded polarized sources can be detected. A secondary limiting factor is the small field-of-view of the instruments, which requires multiple pointings and, hence, an enormous observing time to survey the entire cluster. 


\subsection{Dark Matter: multi-frequency predictions}

From the studies of F. Zwicky in 1933, galaxy clusters are recognized as the largest containers of Dark Matter, the presence of which has been confirmed by gravitational lensing experiments. In particular, lensing studies in the Bullet cluster (Clowe et al. 2006) showed for the first time that the bulk of the matter content in a galaxy cluster was displaced from the hot gas baryonic content, demonstrating the presence of DM. The nature and the properties of the DM are still not determined; a good way to reach this goal would be to detect the electromagnetic signal that DM particles can emit by annihilation or decay (Colafrancesco et al. 2006, Colafrancesco et al. 2011b, Marchegiani \& Colafrancesco 2015).

However, non-thermal emission in the atmospheres of galaxy clusters can have different origins, and manifests itself with a wide range of emission mechanisms and can be then observed over a wide range of frequencies, from radio to gamma rays. For this reason, the detection of genuine DM-produced signal in galaxy clusters appears to be not easy, especially when the baryonic and the DM emissions are co-spatially located. Therefore, a good strategy to search for the DM emission is to study galaxy clusters where the DM and the baryonic halos are spatially offset, like in the case of the Bullet cluster.

It has been shown recently that the complex structure of the radio halo in the Bullet cluster can be used to determine the DM contribution to the radio emission by studying different subhalos corresponding to the observed DM and baryonic dominated regions, and using appropriate non-thermal models to study the different regions of the cluster (Marchegiani \& Colafrancesco 2015). The separation of the radio halo in different subhalos appears to be a promising technique to understand the properties of each subhalo, including the ones produced by DM (see Figures $\square$ and $[$ ). This study has shown that in order to better constrain the properties of the DM subhalos, it is important to perform detailed measures of the radio emission in the regions where the DM halos have their peaks, and that the study of the spatial distribution of the DM subhalos and of the galaxies in the cluster is important to discriminate between their contributions to the overall radio emission. For this reason, it is important to study the properties of DM subhalos in other clusters where the spatial distribution of the radio halo seems to be more similar to the DM distribution than to the X-ray distribution, like in the case of the Coma cluster (e.g. Brown \& Rudnick 2011).

However, the determination of the DM properties (i.e., its nature, mass, composition, annihilation cross section) is still not conclusive, and new studies will be necessary. Another open issue is the effect of small size subhalos, that can amplify the DM annihilation emission by a boost factor between $\sim 30$ and $\sim 1000$, depending on the way it is estimated (Beck \& Colafrancesco 2016). Since the annihilation cross section has been recently constrained to be quite low by Fermi and Planck results (Ackermann et al. 2015; Calore et al. 2015; Ade et al. 2015), it is necessary a high value of the boost factor to have a detectable signal from DM annihilation.

Another open issue related to the DM properties in galaxy clusters is the possible detection of a line at $\sim 43 \mathrm{GeV}$ in the galactic center (Liang et al. 2016), that can be interpreted as produced by DM decay of a particle of similar mass; however, it is still not clear yet if this detection is solid, and in that case if it is produced by DM decay or by other astrophysical sources (e.g. pulsars), therefore pointing to the necessity of performing further studies (e.g. Feng et al. 2016). 


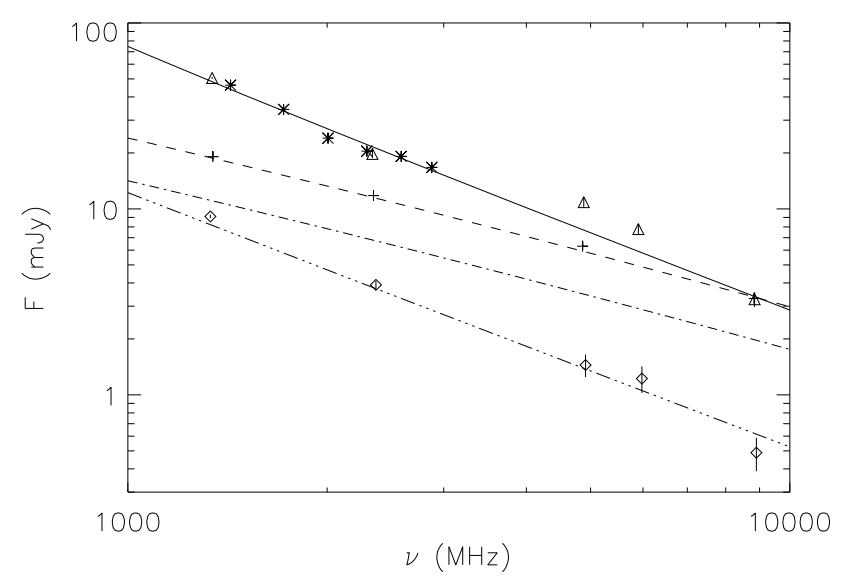

Figure 2: Radio emission from different regions in the Bullet cluster with a Warming Rays model in the Bullet Subcluster (solid line), two DM models the eastern DM region (dashed line) and in the western DM region (dot-dashed line), and a Warming Rays model in the Main Subcluster (three dots-dashed line). Data are from region \# 2 of Liang et al. (2000) (triangles), from the smaller region of Shimwell et al. (2014) (asterisks), from radio source A(L) from Liang et al. (2000) (crosses), and region \# 3 of Liang et al. (2000) (diamonds). Figure from Marchegiani \& Colafrancesco (2015).

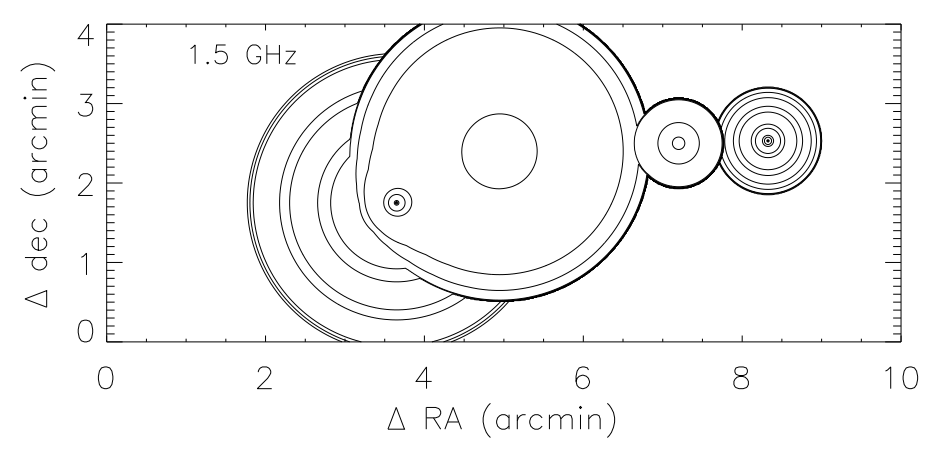

Figure 3: Radio map at $1.5 \mathrm{GHz}$ of the Bullet cluster simulated with the best fit models found in the different regions of the cluster (Fig. (Z). On the axes are the values of the coordinates differences (in arcmin) w.r.t. the origin, fixed at coordinates $(\mathrm{J} 2000) \mathrm{RA}=06: 58: 50$ and DEC $=-55: 59: 00$. Contour levels correspond to: $\left(1 \times 10^{-6}, 5 \times 10^{-6}, 1 \times 10^{-5}\right.$, $5 \times 10^{-5}, 1 \times 10^{-4}, 5 \times 10^{-4}, 1 \times 10^{-3}, 5 \times 10^{-3}, 1 \times 10^{-2}, 5 \times 10^{-2}, 1 \times 10^{-1}, 5 \times 10^{-1}, 1,5,10,50$ and 100) Jy $\operatorname{arcmin}^{-2}$. Figure from Marchegiani \& Colafrancesco (2015).

\subsection{Galaxy feedback: radio-mode, high-E mode, CR injection}

Feedback from active galaxies and AGNs (an important process driving the evolution of galaxy and of the intergalactic medium (IGM), that also regulates SMBH growth and produces a cut-off in the bright end of galaxy luminosity function) has been invoked as one of the main solution to the cooling flow problem in cluster cores preventing overcooling in massive halos. The huge population $(\sim 70 \%)$ of radio loud AGNs that are present in cool-core clusters is an indication for that. The imprint of AGN feedback has been detected in radio and X-ray observations of galaxy 
groups and clusters where the giant radio galaxy lobes are overlaid to the ICM cavities observed in X-ray maps (Birzan et al. 2004). The powerful radio lobes push away the intergalactic gas, induce shock-waves, sound-waves and turbulence into the environment and transfer huge amount of energy from the sub-parsec scale up to Mpc scales in the IGM.

It manifests itself in different modes and with various feedback mechanisms. To explore the radiomode AGN feedback, galaxy groups and clusters must be observed at low radio frequencies $(v \lesssim 1$ $\mathrm{GHz}$, with MeerKAT, LOFAR, GMRT, and the future SKA) where the synchrotron emission from relativistic electrons dominates the continuum radio spectrum of clusters. Deep radio surveys will provide the chance to investigate the evolution of AGN feedback over a wide redshift intervals, and go back in time to see how AGN and central SMBH power would change the dynamics and energetic balances of the ICM. When the central black hole reaches a certain critical mass $\left(10^{12}-\right.$ $10^{13} M_{\odot}$ ), radio mode feedback is triggered, blows out material from the bulge, preventing more accretion (Hickox et al. 2009). While there are a lot of ambiguities in the feeding mechanism toward the center of AGN, gas accretion and black hole spin have been suggested as the main source of energy into the SMBHs. The nature of the gas being accreted remains uncertain. The accretion of cold gas probably brought in through mergers and interactions, or hot accreting gas from the $\mathrm{X}$-ray halos are the main candidates. It has also been suggested that hot and cold mode accretion can explain the dichotomy of radio-mode and radiative-mode (quasar-mode) AGN feedback.

All these arguments show the complexity and tight correlation of AGN feedback, galaxy merger and SMBH growth together with the galaxy evolution within clusters and groups. To break the complexity, it is possible to employ kind of galaxy groups dubbed as "fossils" (Ponman et al. 1994) that are assumed to have no recent major merger for more than $\sim 4$ Gyr in their past history. They are known to be the end-result of galaxy mergers within a group, where $L_{*}$ galaxies merged to form the bright, central elliptical galaxy. The morphology of X-ray emission in fossil galaxy groups is regular and symmetric, indicating the absence of recent major mergers. Therefore, they provide pristine environment to investigate AGN feeding and feedback.

Galaxy and AGN feedback can also feed CRs into the ICM and then induce high-E emission, from hard X-rays to very high-E gamma-rays. During the buoyant rise of bubbles ejected from AGNs and active galaxies CRs diffuse and stream outward producing CR Alfvèn-wave heating. If these bubbles are disrupted, CRs are then injected into the ICM and are caught in a turbulent downdraft that is possibly excited by the rising bubbles (see, e.g., Pfrommer 2013). CRs then escape and diffuse in the ICM, thus producing both heating of the ICM and high-E emission by interacting with the ambient protons. The high-E emission triggered by this AGN feedback is expected with specific spatial and spectral features that can be separated from the underlying highE emission due to the truly diffuse population of CRs in the cluster atmosphere (Colafrancesco, Marchegiani and Giommi 2010). Detecting such high-E emission patterns will have a crucial impact on both cluster physics and their galaxy population evolution.

\section{A multi-frequency perspective}

These controversies related to the non-thermal phenomena in galaxy clusters can be solved by adopting a multi-band approach, where information coming from different energy bands can be 
combined to better constrain the clusters' properties. In the following, we discuss some recent and future progresses foreseen in several energy bands.

\subsection{The millimeter range}

The SZE is the spectral distortion of the CMB by inverse Compton scattering from the electrons residing in the cluster atmospheres (see, e.g., Sunyaev \& Zel'dovich 1972). It has been demonstrated (Colafrancesco et al. 2003) that a relativistically correct calculation of the SZE is necessary to study the properties of both thermal and non-thermal electrons in different kinds of extragalactic objects. In fact, relativistic corrections allow to derive the properties of the thermal gas from the spectral shape of the SZE, and to study the SZE produced by relativistic electrons.

Recently, the study of the SZE spectrum up to high frequencies allowed to estimate the properties of the non-thermal electrons in the Bullet cluster (Colafrancesco et al. 2011a, Marchegiani and Colafrancesco 2015), and to derive that the gas temperature distribution along the line of sight is strongly inhomogeneous (Prokhorov \& Colafrancesco 2012). Specifically, the SZE in the Main Cluster of the Bullet cluster points towards a non-thermal electrons population with a very low minimum momentum, therefore suggesting a primary origin for these electrons (Marchegiani \& Colafrancesco 2015; see Figure (1). Therefore, the SZE is a powerful tool to probe the properties of thermal and non-thermal electrons in galaxy clusters and other extragalactic objects like radiogalaxies halos. To obtain better and more precise results, it is important to perform spectral studies of the SZE over a wide frequency range, and high frequencies $(v \gtrsim 400 \mathrm{MHz})$ are particularly important to detect non-thermal signatures in the SZE spectrum. Space-borne instruments like Millimetron (Kardashev et al. 2015) can have a great relevance in performing these studies.

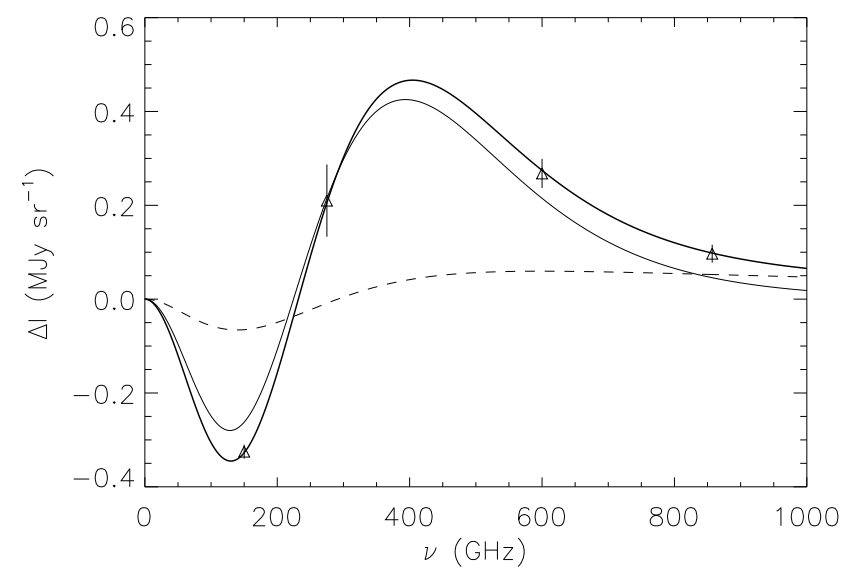

Figure 4: SZE in the Bullet cluster, with a thermal effect in the Main Subcluster (solid line), a non-thermal effect minimum electrons momentum $p_{1}=1$ (dashed line), and the sum of the two components (thick line). Data at 150 and $275 \mathrm{GHz}$ are taken with ACBAR (Gomez et al. 2004), and data at 600 and $857 \mathrm{GHz}$ with Herschel-SPIRE (Zemcov et al. 2010). Figure from Marchegiani \& Colafrancesco (2015).

In this context, the SZE can be considered as a new and powerful probe of high-E processes in galaxy clusters for several reasons. First, it is a scattering/diffusion effect and therefore it is 
not depending on the distance of the source, whereas measures of integrated flux in other spectral bands (e.g. X-rays or gamma-rays) are strongly affected by their distance. In addition, the SZE is linearly proportional to the electrons density, and therefore it can be detected also in regions with low density, like the peripheral regions of galaxy clusters, and it is particularly suitable to detect the non-thermal electrons in radio halos, relics, radiogalaxy lobes and bubbles of relativistic plasma inside the ICM. Since the SZE is not depending on the magnetic field intensity, these measure are able to constrain the electrons density, and thus derive the magnetic field separately when combined with radio observations. This information is important to constrain the amount and evolution of the CRs in galaxy clusters, to understand their origin, as well as the properties of shocks and other non-thermal processes occurring during the cluster evolution. Finally, an instrument operating in radio bands with high angular resolution and sensitivity like the SKA can also detect the SZE at low frequencies, and distinguish the SZE from other sources of radio emission in the cluster (compact or diffuse), allowing to perform these studies and to derive important information on the physics of galaxy clusters and of the cosmological radio backgrounds (Colafrancesco et al. 2015, 2016).

\subsection{X-rays}

Detailed X-ray studies of the ICM provide information about the properties of the thermal gas and the non-thermal feedback on it. It is, in fact, possible to derive information on the properties of shock fronts in merging cluster, and derive information on the properties of non-thermal electrons accelerated by these phenomena (e.g. Uchida et al. 2016). Also, it is possible to derive information on the properties of bubbles of relativistic plasma in the ICM (Fabian et al. 2000).

The detection of galaxy clusters in frequency bands close to X-rays, i.e. extreme Ultra-Violet and hard X-rays, is still debated, both regarding the effective detection and the physical origin of the emission. For the EUV, an emission in excess with respect to the thermal gas has been confirmed in Coma and Virgo (Bowyer et al. 1999, Berghofer et al. 2000), but it is not clear if this emission is thermal (Lieu et al. 1996) or due to the ICS of non-thermal electrons (Sarazin \& Lieu 1998); Bowyer et al. (2004) suggested a possible origin from ICS of secondary electrons, but this possibility has been excluded by Marchegiani et al. (2007), because it requires a quantity of nonthermal protons with pressure bigger than the thermal one, and would predict an excess of gamma rays not observed by EGRET. For the HXR, the initial claims of detection (e.g. Fusco-Femiano et al. 1999) have been questioned (e.g. Rossetti \& Molendi 2004), and at present time only upper limits are available (e.g. Ajello et al. 2009, Gastaldello et al. 2015).

ICS in the HXR band is expected from the extended radio halos and relics in clusters with different spectral shape and spatial distribution. If the ICS is produced by the same electrons producing the radio emission, we can expect an HXR emission with the same spectral index than the radio one (e.g. Longair 1994), but dependent only on the electrons density. Therefore, we would expect a more extended emission than the radio one, that instead is more concentrated in the regions where the magnetic field is higher. Also a possible change in the slope of the electrons spectrum (that in turn depends on the properties of the acceleration source and energy losses) would be observed at a frequency not depending on the magnetic field intensity, allowing to constrain the properties of the electrons, of the acceleration mechanisms, and of the energy losses in the ICM. Therefore, a detection with Astro-H2 of the ICS produced by non-thermal electrons would be extremely important, because it would allow to determine the spectrum and the density of non- 
thermal electrons independently from radio observations, and to obtain an estimate of the value of the magnetic field (e.g. Colafrancesco et al. 2005).

\subsection{Soft gamma rays}

At present, galaxy clusters are unexplored in the soft Gamma Ray (SGR) band (i.e., 0.1$10 \mathrm{MeV}$ ). Here, we will estimate if a forthcoming mission like Astro-H2/SGD and the proposed missions AstroMeV and ASTROGAM will be able to detect an emission from the diffuse nonthermal components of galaxy clusters.

In principle, two mechanisms can produce emission in the SGR: ICS of the CMB (and other background photon fields) from high-energy electrons (with $\mathrm{E}$ around $10 \mathrm{GeV}$ ), and non-thermal bremsstrahlung with the ICM nuclei from mildly relativistic electrons (with energy of order $\mathrm{MeV}$ ). The first mechanism is strongly constrained by the present HXR upper limits in galaxy clusters (e.g. Ajello et al. 2009) and the steep spectrum that these electrons should have, in the light of the steepening of the high-frequency part of the radio halo spectrum (e.g. Thierbach et al. 2003). The second mechanism is difficult to observe, because of the inefficiency of the non-thermal bremsstrahlung compared to the high rate of energy losses due to the Coulomb interactions in the ICM; therefore, we expect that the non-thermal bremsstrahlung could be observed only for a very short time compared to the lifetime of the cluster (see also discussion in Petrosian 2001).

However, the possibility to constrain the low-energy part of the electrons spectrum, for which at present we have only indications from the possible detection of the non-thermal SZE (e.g. Colafrancesco, Marchegiani \& Buonanno 2011a for the case of the Bullet cluster), makes interesting to compare the predicted emission with the properties of the planned SGR instruments.

The secondary models, both of hadronic and Dark Matter origin, do not predict a strong emission in the SGR, because the rate of low-E electrons production is small compared to the loss rate, and the present upper limits of Fermi-LAT set strong constraints on the density of CR protons and on the DM annihilation cross section (see discussion in the next section). Figure $\square$ shows an example of the SED we expect from these models. Also re-acceleration models based on stochastic re-acceleration by turbulences in the ICM (e.g. Brunetti et al. 2004) do not predict a strong emission in the SGR, because these processes are mainly efficient for electrons with Lorentz factor of $\gamma \sim 10^{3}-10^{4}$ (see Fig.14 in Takahashi et al. 2013).

Therefore the only possibility to detect the non-thermal bremsstrahlung from low-E electrons is for a very short time (of the order of $10^{7}-10^{8} \mathrm{yrs}$ ) from primary electrons (see also Petrosian 2001). Furthermore, a very high injection rate is required to obtain a high density of low-E electrons in order to maximize their non-thermal bremsstrahlung emission. To check this possibility, we assume in a Coma-like cluster a source of cosmic ray electrons with a power-law spectrum $Q_{e}(\gamma) \propto$ $\gamma^{-s_{e}}$ with $s_{e}=2.7$ and a power of $10^{45} \mathrm{erg} \mathrm{s}^{-1}$, that is of the order of the maximum energy input we should expect as a result of different processes injecting or accelerating particles in galaxy clusters (e.g. Berezinski et al. 1997), active for $2 \times 10^{9} \mathrm{yrs,}$, and we calculate the evolution of the electron spectrum after the beginning and after the end of the injection process (e.g. Sarazin 1999). We consider only the effect of the injection term and the loss term, neglecting the diffusion and the presence of possible re-acceleration effects. The SGR emission produced by ICS and nonthermal bremsstrahlung is shown in Fig.6. As we can see, we do not expect that an emission can be observed also with the proposed missions AstroMeV and ASTROGAM. We expect, instead, 


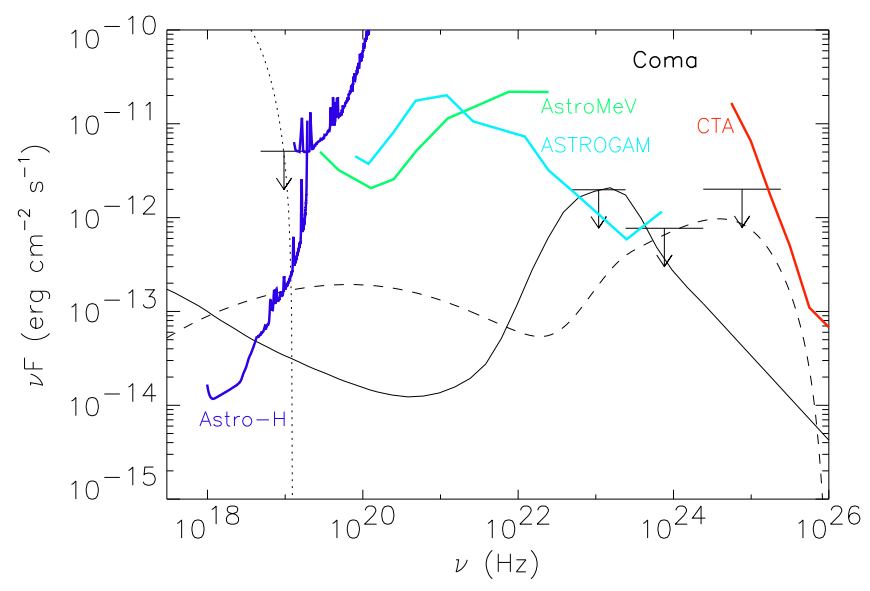

Figure 5: Hard X-ray and gamma-ray emission in the Coma cluster produced by the sum of inverse Compton scattering, non-thermal bremsstrahlung emissions and $\pi^{0}$ decay for secondary electrons models from hadronic collisions (solid line) and Dark Matter annihilation for $M_{\chi}=500 \mathrm{GeV}$ and $b \bar{b}$ composition (dashed line). The maximum level of emission allowed by the present Fermi-LAT upper limits is shown. We plot the upper limits of NuSTAR (Gastaldello et al. 2015) and Fermi-LAT (Ackermann et al. 2010), and the expected sensitivities of Astro-H (HXI and SGD) for $100 \mathrm{ks}$ of time integration (from http://astro-h.isas.jaxa.jp/researchers/sim/sensitivity.html), AstroMev for 5 yrs of survey (from http://astromev.in2p3.fr/sites/default/files/Takahashi-COCOTE_sens.jpg), ASTROGAM for an effective exposure of 1 yr (from http://astrogam.iaps.inaf.it/scientific_instrument.html) and CTA for 1000 hrs (from Funk \& Hinton 2013). The dotted line shows the thermal emission produced by the hot ICM in Coma.

that the ICS emission can be observed with an experiment like Astro-H/HXI, but in this case it is necessary to separate the contribution of the thermal emission at high energy.

\subsection{High-energy gamma-rays}

The new Fermi-LAT upper limits on galaxy clusters allow to set new limit on the density of cosmic rays protons and on the cross sections of annihilation of Dark Matter.

For hadronic secondary electrons models, it is well known that the decay of neutral pions produced in hadronic interactions produces an emission in the gamma-rays with a peak located around $E \sim m_{\pi} / 2 \sim 70 \mathrm{MeV}$; therefore an instrument like the Fermi-LAT can constrain the properties of non-thermal protons in galaxy clusters (e.g. Colafrancesco \& Blasi 1998, Marchegiani et al. 2007). However, the situation is still not clear because the limits found by the Fermi Collaboration are changing by changing the procedures of data analysis. For example from the limit on the Coma cluster in Ackermann et al. (2014), obtained from the analysis of four years of observations with Fermi-LAT, it is possible to derive for the ratio between the non-thermal protons pressure and the thermal one a very small upper limit of the order of $4 \%$, whereas by using the limit obtained with the Pass- 8 analysis of six years of observations (Ackermann et al. 2016), an upper limit of $13 \%$ is derived. Therefore, new and complete data analysis of Fermi data will be necessary to derive strong consequences on the physics of cosmic rays in galaxy clusters. Anyway, it appears evident that the amount of cosmic rays is much less than what was previously found in numerical simulations (e.g. Ryu et al. 2003), and these studies need to be improved (Vazza et al. 2016). 


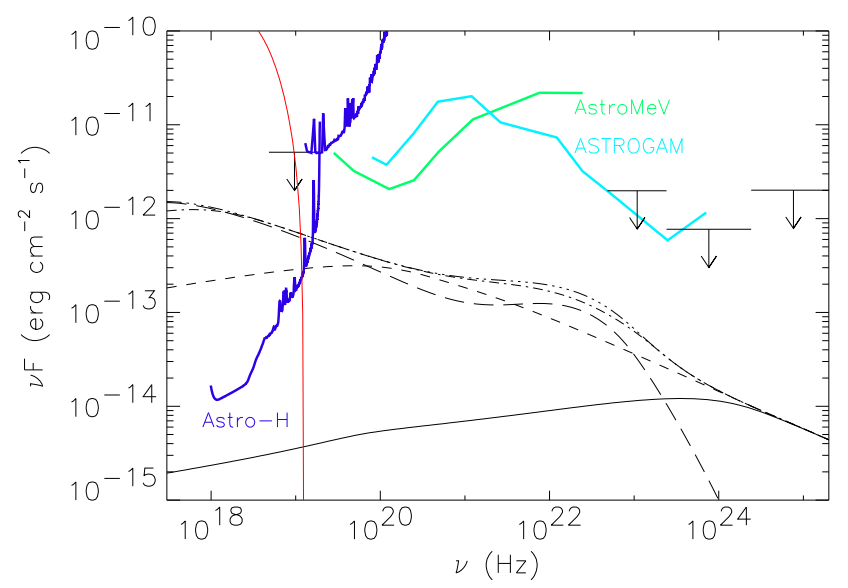

Figure 6: Evolution of the hard X-ray and gamma-ray emission in the Coma cluster given by the sum of inverse Compton scattering and non-thermal bremsstrahlung emissions for an electrons population resulting from a source with a power-law spectrum with spectral index 2.7 and power of $10^{45} \mathrm{erg} \mathrm{s}^{-1}$ active for $2 \times 10^{9} \mathrm{yrs}$, after $10^{6}$ (solid line), $10^{8}$ (dashed), $10^{9}$ (dot-dashed), $2 \times 10^{9}$ (three dots-dashed) yrs from the beginning of the injection, and $2 \times 10^{7}$ yrs after the end of the injection. The red line shows the thermal emission produced by the hot gas in Coma.

The limits that can be derived on the Dark Matter annihilation cross section depend strongly on the assumed properties of the Dark Matter particles; in general, more constraining limits are obtained for mass of Dark Matter in the energy range of observations of Fermi-LAT, whereas limits are less strong for high values of the neutralino mass $\left(M_{\chi}>500 \mathrm{GeV}\right)$, because in this case the peak of the emission is located outside of the energy band of Fermi.

This last consideration suggests the possibility to constrain the properties of the Dark Matter by observing galaxy clusters with the next generation of Cerenkov instruments like H.E.S.S. II and CTA. In fact, if Dark Matter particles have mass of the order of $\mathrm{TeV}$, an emission in the range of Cerenkov telescopes should be produced by Dark Matter annihilation, whereas gamma-ray emission of hadronic origin is not expected to be strong in this band (see Fig.D). This fact makes very interesting the study of galaxy clusters with the next generation of Cerenkov telescopes.

\section{Conclusions}

Many aspects of the high-E phenomena in galaxy clusters still need to be clarified. In particular, the spectrum and abundance of cosmic rays (both electrons and protons), the process driving to the formation of the radio halo and relics, the properties of magnetic fields, the nature and the properties of the Dark Matter, the role of galaxies of different types (normal, starburst, radio galaxies and AGNs) on the physics of the ICM, even if progress has been made in last years, still show open issues from both experimental and theoretical point of views.

New theoretical and observational studies, focusing on constraining spectral and spatial properties of non-thermal emissions, especially in radio, microwave, X-ray and gamma-ray bands, will help in solving these issues. We expect that information derived with instruments like SKA, Mil- 


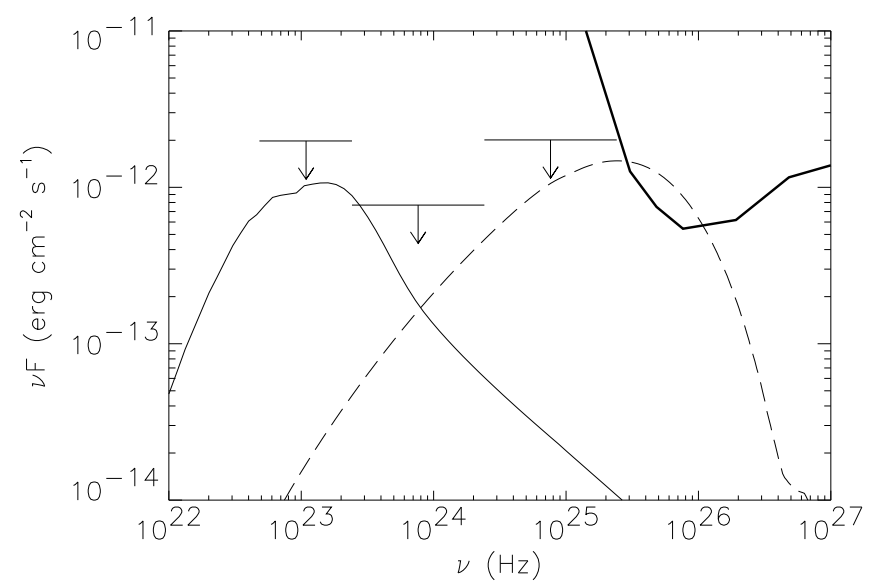

Figure 7: Gamma-ray emission in the Coma cluster for a hadronic secondary model with non-thermal proton spectrum with spectral index 2.7 (solid line) and a Dark Matter model for a neutralino with $M_{\chi}=3000 \mathrm{GeV}$ (dashed line). Both the models are normalized to the upper limits of Fermi. We show the upper limits of Fermi (Ackermann et al, 2014) and the sensitivity of H.E.S.S. II for $50 \mathrm{hrs.}$

limetron, Astro-H2, and CTA, as well as the full set of Fermi-LAT data, once combined, will strongly contribute to our understanding of physics of galaxy clusters.

\section{Acknowledgments}

This work is based on the research supported by the South African Research Chairs Initiative of the Department of Science and Technology and National Research Foundation of South Africa (Grant No 77948). P.M. acknowledges support from the DST/NRF SKA post-graduate bursary initiative.

Disclaimer: any opinion, finding and conclusion or recommendation expressed in this material is that of the author(s) and the NRF does not accept any liability in this regard.

\section{References}

[1] Ackermann, M. et al., 2010, ApJL, 717, L71

[2] Ackermann, M., et al., 2014, Phys. Rev. D, 89, 042001

[3] Ackermann M. et al., 2015, Physical Review Letters, 115, 231301

[4] Ackermann M. et al., 2016, ApJ, 819, 149

[5] Ade P.A.R., et al., 2015, A\&A, in press, arXiv:1502.01589

[6] Ajello, M. et al., 2009, ApJ, 690, 367

[7] Basu, K. 2012, MNRAS, 421, L112

[8] Bayer-Kim C.M. et al., 2002, MNRAS, 337, 938

[9] Beck G., Colafrancesco S., 2016, JCAP, 05, 013 
[10] Berezinsky, V.S., Blasi, P. \& Ptuskin, V.S., 1997, ApJ, 487, 529

[11] Berghöfer, T.W., Bowyer, S. \& Korpela, E. 2000, ApJ, 535, 615

[12] Bernardi, G., et al., 2016, MNRAS, 456, 1259

[13] Bird J., Martini P., Kaiser C., 2008, ApJ, 676, 147

[14] Birzan, L., et. al., 2004, 'A Systematic Study of Radio-induced X-Ray Cavities in Clusters, Groups, and Galaxies' ApJ, 607, 800

[15] Blasi, P. \& Colafrancesco, S. 1999, Astroparticle Physics, 12, 169

[16] Bonafede, A., Dolag, K., Stasyszyn, F., Murante, G., Borgani, S., 2011, MNRAS, 418, 2234

[17] Bonafede, A., et al., 2015, MNRAS, 454, 3391

[18] Bowyer, S., Berghöfer, T.W. \& Korpela, E.J., 1999, ApJ, 526, 592

[19] Bowyer, S., Korpela, E.J., Lampton, M. \& Jones, T.W., 2004, ApJ, 605, 168

[20] Brown S., Rudnick L., 2011, MNRAS, 412, 2

[21] Brüggen, M., 2013, MNRAS, 436, 294

[22] Brunetti, G., Setti, G., Feretti, L. \& Giovannini, G. 2001, MNRAS, 320, 365

[23] Brunetti, G., Blasi, P., Cassano, R. \& Gabici, S., 2004, 350, 1174

[24] Brunetti G, Cassano, R., Dolag, K. and Setti, G. 2009, A\&A, 507, 661

[25] Brunetti, G., et al., 2012, MNRAS, 426, 956

[26] Calore F., Cholis I., McCabe C., Weniger C., 2015, PhRvD, 91, 063003

[27] Cassano, R., et al., 2013, ApJ, 777, 141

[28] Clowe, D., et al., 2006, ApJ, 648, L109

[29] Colafrancesco, S., 1999, MmSAI, 70, 851

[30] Colafrancesco, S. 2010, in ASTROPHYSICS AND COSMOLOGY AFTER GAMOW: Proceedings of the 4th Gamow International Conference on Astrophysics and Cosmology After Gamow and the 9th Gamow Summer School "Astronomy and Beyond: Astrophysics, Cosmology, Radio Astronomy, High Energy Physics and Astrobiology”. AIP Conference Proceedings, Volume 1206, pp. 5-26

[31] Colafrancesco, S., 2013, invited review at the Frascati Meeting 'Frontier objects between particle physics and astrophysics', 2012, Vulcano (Italy), Acta Polytech., 53, 560

[32] Colafrancesco, S. \& Blasi, P., 1998, Astroparticle Physics, 9, 227

[33] Colafrancesco S., \& Mele B., 2001, ApJ 562, 24

[34] Colafrancesco, S., \& Marchegiani, P., 2008, A\&A, 484, 51

[35] Colafranceso, S. \& Marchegiani, P., 2014, A\&A, 562, L2

[36] Colafrancesco, S., Marchegiani, P., \& Palladino, E., 2003, A\&A, 397, 27

[37] Colafrancesco, S., Dar, A. \& De Rújula, A., 2004, A\&A, 413, 441

[38] Colafrancesco, S., Marchegiani, P. \& Perola, G.C., 2005, A\&A, 443, 1

[39] Colafrancesco S., Profumo S., Ullio P., 2006, A\&A, 455, 21 
[40] Colafrancesco S., Profumo S., Ullio P., 2007, PhRvD, 75, 023513

[41] Colafrancesco, S., Marchegiani, P. and Giommi,P. 2010, A\&A, 519, 82

[42] Colafrancesco, S., Marchegiani, P. \& Buonanno, R., 2011a, A\&A, 527, L1

[43] Colafrancesco, S., et al., 2011b, A\&A, 527, A80

[44] Colafrancesco, S., Emritte, M.S., Mhlahlo, N. \& Marchegiani, P., 2014, A\&A, 566, A42

[45] Colafrancesco, S., Emritte, M.S \& Marchegiani, P., 2015, JCAP, 05, 006

[46] Colafrancesco, S., Marchegiani, P., Emritte, M.S., 2016, A\&A, in press, arXiv:1607.07723

[47] Dennison, B., 1980, ApJ, 239, L93

[48] Dolag, K., Bykov, A. M., Diaferio, A., 2008, Space Science Reviews, 134, 311

[49] Donahue M. et al., 2005, ApJ, 630, L13

[50] Dwarakanath, K.S. \& Nath, B.B., 2006, ApJ, 653, L9

[51] Fabian A. C., et al., 2000, MNRAS, 318, L65

[52] Fabian, A.C., \& Sanders, J.S., 2007, proceedings of "Heating vs Cooling in Galaxies and Clusters of Galaxies", August 2006, Garching, p.65 (astro-ph/0612426)

[53] Fabian, A.C., Sanders, J.S., Taylor, G.B., et al., 2006, MNRAS, 366, 417

[54] Feng, L., et al., 2016, Phys. Rev. D, in press, arXiv:1608.04056

[55] Feretti, L., Giovannini, G., Govoni, F., Murgia, M., 2012, A\&ARv, 20, 54

[56] Ferrari, C., et al., 2015, Proceedings of Advancing Astrophysics with the Square Kilometre Array (AASKA14). 9 -13 June, 2014. Giardini Naxos, Italy, p.75

[57] Fujita, Y. \& Suzuki, T.K., 2005, ApJ, 630, L1

[58] Funk, S. \& Hinton, J.A., 2013, APh, 43, 348

[59] Fusco-Femiano, R., Dal Fiume, D., Feretti, L., Giovannini, G., Grandi, P., Matt, G., Molendi, S. \& Santangelo A., 1999, ApJ, 513, L21

[60] Gastaldello, F. et al., 2015, ApJ, 800, 139

[61] George, L.T., et al., 2015, MNRAS, 451, 4207

[62] Giovannini, G., Tordi, M., Feretti, L., 1999, New Astronomy, 4, 141

[63] Gitti, M., Brunetti, G., Setti, G., 2002, A\&A, 386, 456

[64] Gomez, P. et al. 2004, in Plasmas in the laboratory and in the Universe: New Insights and New Challenges., AIP Conference Proceedings, 703, 361

[65] Govoni, F. \& Feretti, L. 2004, International Journal of Modern Physics D, 13, 1549

[66] Govoni, F., et al., 2006, A\&A, 460, 425

[67] Govoni, F., et al., 2012, A\&A, 545, A74

[68] Govoni, F., et al., 2015, Proceedings of Advancing Astrophysics with the Square Kilometre Array (AASKA14). 9 -13 June, 2014. Giardini Naxos, Italy, p.105

[69] Heinz, S., Brüggen, M., Young, A., Levesque, E., 2006, MNRAS, 373, L65 
[70] Hickox, R.C., et al., 2009, ApJ, 696, 891

[71] Huber, B., et al., 2013, A\&A, 560, A64

[72] Jaffe, W.J. 1977, ApJ, 212, 1

[73] Johnston-Hollitt, M., et al., 2015, Proceedings of Advancing Astrophysics with the Square Kilometre Array (AASKA14). 9 -13 June, 2014. Giardini Naxos, Italy, p.92

[74] Kardashev, N.S. et al., 2015, arXiv:1502.06071

[75] Knowles, K., et al., 2016, MNRAS, 459, 4240

[76] Liang, H., Hunstead, R.W., Birkinshaw, M., \& Andreani, P., 2000, ApJ, 544, 686

[77] Liang, Y.F., et al., Phys. Rev. D, 2016, 93, 103525

[78] Lieu, R., Mittaz, J., Bowyer, S., Breen, J., Lockman, F., Murphy, E. \& Hwang, C.-Y., 1996, Science, 274, 1335

[79] Longair, M. 1994, High Energy Astrophysics, Cambridge University Press

[80] Marchegiani P., Colafrancesco S., 2015, MNRAS, 452, 1328

[81] Marchegiani, P., Perola, G.C. \& Colafrancesco, S., 2007, A\&A, 465, 41

[82] McNamara, B. and Nulsen, ... 2007, ...

[83] Petrosian, V., 2001, ApJ, 557, 560

[84] Petrosian, V. \& East, W.E., 2008, ApJ, 682, 175

[85] Pfrommer, C. 2013, ApJ, 779, 10.

[86] Ponman, T.J., et al., 1994, Nature, 369, 462

[87] Prokhorov, D. A. \& Colafrancesco, S., 2012, MNRAS, 424, L49

[88] Rossetti, M. \& Molendi, S., 2004, A\&A, 414, L41

[89] Ryu, D., Kang, H., Hallman, E. \& Jones, T.W. 2003, ApJ, 593, 599

[90] Sanders, J.C. \& Fabian, A.C., 2007, MRAS, 381, 1381

[91] Sarazin C.L., 1988, 'X-ray emission from clusters of galaxies', Cambridge University Press

[92] Sarazin, C.L. 1999, ApJ, 520, 529

[93] Sarazin, C.L. \& Lieu, R., 1998, ApJ, 494, L177

[94] Shimwell, T.W., et al., 2014, MNRAS, 440, 2901

[95] Sijacki, D. \& Springel, V., 2006 MNRAS, 366, 397

[96] Springel V. et al., 2005, Nature, 435, 629

[97] Sunyaev R.A., Zel'dovich Y.B., 1972, A\&A, 20, 189

[98] Takahashi, T., Uchiyama, Y., and Stawarz, L., 2013, Astroparticle Physics, 43, 142

[99] Thierbach, M., Klein, U. \& Wielebinski, R. 2003, A\&A, 397, 53

[100] Tribble, P.C., 1993, MNRAS, 263, 31

[101] Uchida, Y., et al., 2016, PASJ, 68, S20 
[102] Vacca, V., et al., 2012, A\&A, 540, A38

[103] Vazza, F., Brüggen, M., 2014, MNRAS, 437, 2291

[104] Vazza, F., et al., 2016, MNRAS, 459, 70

[105] Vernaleo, J.C. \& Reynolds, C.S., 2005, AAS, 207, 5503

[106] Vernaleo, J.C. \& Reynolds, C.S., 2006, ApJ, 645, 83

[107] Vernaleo, J.C. \& Reynolds, C.S., 2007, ApJ, 671, 171

[108] Wolfe, B., \& Melia, F., 2006, ApJ, 638, 125

[109] Zandanel, F., Pfrommer, C., Prada, F., 2014, MNRAS, 438, 124

[110] Zemcov, M. et al., 2010, A\&A, 518, L16 